\title{
Bilateral internal carotid artery occlusion with compensatory perfusion in a $\mathrm{HIV}$-infected patient
}

\author{
SM Gowan, LM Ford, IN Human, V Goolab \\ Division of Neurosurgery, Department of Neurosciences, University of the Witwatersrand, Johannesburg, South Africa
}

Corresponding author, email: gowan.neurosurg@gmail.com

\begin{abstract}
Summary
HIV infection occlusive arteriopathies may result in neurological symptoms. We report a case of bilateral complete occlusion of the extracranial portions of the internal carotid arteries in a HIV+ve patient who presented with a syncopal episode due to intraventricular haemorrhage. Compensatory blood flow from the posterior cerebral circulation via the circle of Willis resulted in small telangiectatic vessels arising from the posterior cerebral circulation which probably accounted for this rare haemorrhagic complication of an occlusive arteriopathy.

Keywords: HIV, occlusive arteriopathy, internal carotid artery
\end{abstract}

\section{Case report}

A 41-year-old female patient of African heritage presented with a single syncopal episode. Neurological examination on admission noted the absence of any motor, sensory or cognitive deficits. This previously diagnosed HIV+ve patient had been receiving highly active antiretroviral therapy (HAART) treatment and long-term antihypertensive medications. A CT brain scan displayed an intraventricular haemorrhage (Figure 1) and formal angiography revealed occlusion of both of the extracranial internal carotid arteries with compensatory blood flow from the posterior cerebral vasculature and associated collateral small vessel formation (Figure 2). Evidence of a coagulopathy was absent on blood investigations and the CD4 count was 630 cells/ $\mu \mathrm{L}$. No further syncopal episodes or neurological deterioration occurred. No significant family history or other comorbid conditions were noted. She was managed conservatively with surgical revascularisation deferred unless clinical evidence of cerebral hypo-perfusion was noted at a later stage. At the most recent follow-up visit, six months after her original admission to our facility, the patient reported no seizures or neurological deficits and was able to perform her normal daily activities.

\section{Discussion}

In 2006, it was reported that of the total South African population of 48 million people, 5.4 million were infected with HIV. ${ }^{1}$ It is well recognised that HIV infection predisposes patients to a multitude of neurological conditions, including cerebrovascular disease. ${ }^{2}$ Systemically, HIV-related vasculopathies have a predilection for medium and large vessels, and its pathogenesis is attributable to direct viral invasion of vascular cells, injury caused by viral proteins, the effects of opportunistic infections, chronic inflammation or adverse effects of anti-retroviral medications. ${ }^{1,2}$
Histological findings in occlusive HIV-related vascular lesions consisted of changes in the media (including scattered chronic inflammatory cell infiltrate, focal calcification, smooth muscle injury and fibrosis) along with fragmentation of the internal elastic lamina.,3 Separate animal studies, using HIV-1 provirus infected transgenic mice, and simian immunodeficiency virus (SIV)-infected rhesus monkeys, demonstrated occlusive arteriopathies characterised by intimal hyperplasia with associated fibrosis in the latter. ${ }^{2,3}$ Endothelial dysfunction is believed to be secondary to continued viral infection and injury from viral proteins, leading to release of interleukin 1 (IL1), interleukin 6 (IL6), interleukin 8 (IL8) and tumour necrosis factor- $\alpha$ (TNF- $\alpha){ }^{3}$ Even though HIV is not specifically vasculotropic, vascular cells are continually and systemically exposed to viral proteins, inflammatory cells and cytokines. ${ }^{2}$

Evidence suggests a contributory role of HAART in cerebrovascular disease, with protease inhibitors being linked to dyslipidaemia and insulin resistance. ${ }^{4}$ Immune activation and reconstitution with associated upregulation of pro-inflammatory cytokines following HAART initiation can also result in endothelial dysfunction and thrombosis. ${ }^{2,3}$

CD4 counts were significantly higher in patients with extracranial cerebrovascular disease (as was the case in our patient) when compared to those with exclusively intracranial involvement. ${ }^{1}$ This suggests differing mechanisms between intra- and extracranial lesion formation, potentially with a greater emphasis on immune activation in the latter. ${ }^{5}$

Other occlusive arteriopathies involving the internal carotid arteries, like Moyamoya syndrome, are characterised by the development of compensatory collateral vasculature comprising of fragile small vessels, often the source of intracranial haemorrhages. ${ }^{6}$ Angiography in our case report displayed a similar compensatory telangiectasia arising from the posterior cerebral circulation. We postulate that 


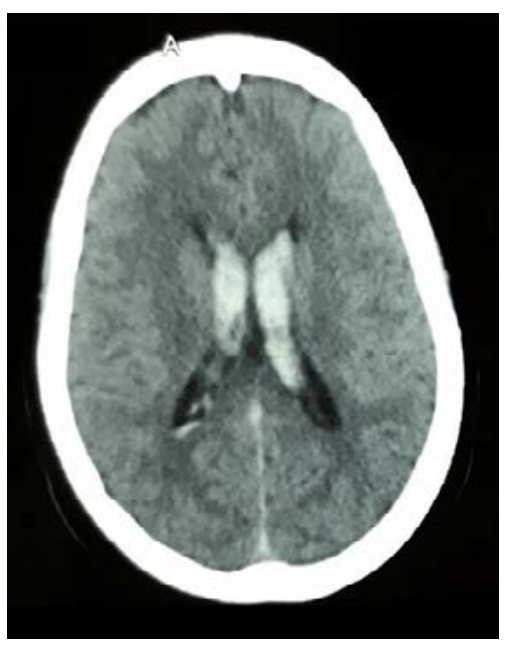

Figure 1: CT brain scan showing vascular enhancement in the ventricles typical of intraventricular haemorrhage

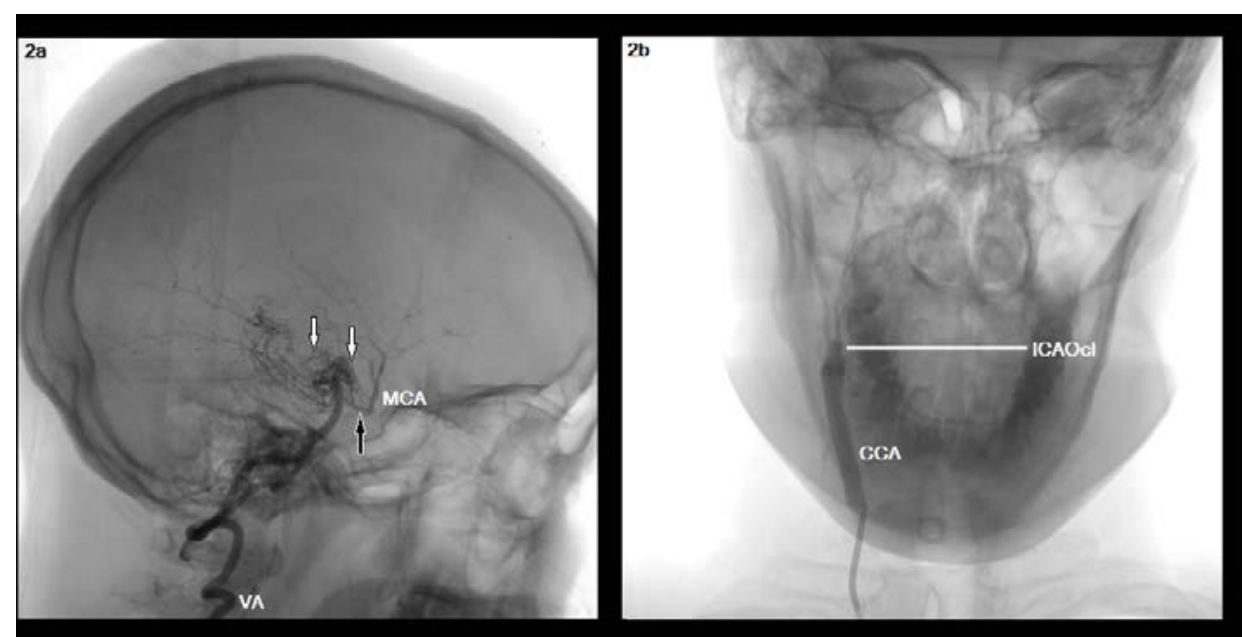

Figure 2: Cerebral angiography 2a) lateral view: compensatory perfusion from posterior cerebral circulation with associated telangiectatic small vessels; 2b) anterior view: complete occlusion of bilateral internal carotid arteries

$M C A$ - middle cerebral artery; VA - vertebral artery; $C C A$ - common carotid artery; ICAocl - level of internal carotid artery occlusion; black arrow - collateral blood flow through posterior communicating artery; white arrows - telangiectasia the fragility of these small vessels may account for the intracranial haemorrhage in our patient.

\section{Conclusion}

With the high global prevalence of HIV-related conditions and the growing number of patients receiving antiretroviral treatment, occlusive arteriopathies in these patients are gaining increasing clinical relevance. This is a condition of multi-factorial pathogenesis and may produce unusual presenting features and radiological findings, as demonstrated by this case report.

\section{Ethics declaration}

Informed consent for collection of data was obtained from the patient and the research was approved by the Human Research Ethics Committee, University of the Witwatersrand.

\section{Conflict of interest}

The authors declare no conflict of interest.

\section{Funding source}

No funding source to be declared.

\section{REFERENCES}

1. Tipping B, De Villiers L, Wainwright $\mathrm{H}$, et al. Stroke in patients with human immunodeficiency virus infection. J
Neurol Neurosurg Psychiatry. 2007 Dec; 78(12):1320-4. Available from: http://dx.doi.org/10.1136/jnnp.2007.116103. PMID: 17470469.

2. Edwards NJ, Grill MF, Choi HA, et al. Frequency and Risk Factors for Cerebral Arterial Disease in a HIV/AIDS Neuroimaging Cohort. Cerebrovasc Dis. 2016;41(3-4):170-6. Available from: http://dx.doi.org/10.1159/000442755. PMID: 26751784.

3. Pillay B, Ramdial PK, Naidoo DP. HIV-associated largevessel vasculopathy: a review of the current and emerging clinicopathological spectrum in vascular surgical practice. Cardiovasc J Afr. 2015 Mar-Apr;26(2):70-81. Available from: http://dx.doi.org/10.5830/CVJA-2015-017. PMID:25940120.

4. Regina G, Impedovo G, Angiletta D, et al. Surgical experience with carotid stenosis in young HIV-1 positive patients under antiretroviral therapy: an emerging problem? Eur J Vasc Endovasc Surg. 2005 Feb;29(2):167-70. Available from: http://dx.doi.org/10.1016/j.ejvs.2004.09.017. PMID: 1564972.

5. Connor M. Stroke in patients with human immunodeficiency virus infection. J Neurol Neurosurg Psychiatry. 2007 Dec;78(12):1291. Available from: http://dx.doi.org/10.1136/ jnnp.2007.122416. PMID: 18024688.

6. Smith ER, Scott RM. Moyamoya: epidemiology, presentation, and diagnosis. Neurosurg Clin N Am. 2010 Jul;21(3):543-51. Available from: http://dx.doi.org/10.1016/j.nec.2010.03.007. PMID: 20561502. 